\title{
CONGRUENCES FOR THE COEFFICIENTS OF THE MODULAR INVARIANT $j(\tau)$
}

\section{HANS-FREDRIK AAS}

1.

The modular invariant $j(\tau)$ is defined by

$$
j(\tau)=x^{-1} \prod_{1}^{\infty}\left(1-x^{n}\right)^{-24}\left(1+240 \sum_{1}^{\infty} \sigma_{3}(n) x^{n}\right)^{3}, \quad x=e^{2 \pi i \tau},
$$

where

$$
\sigma_{k}(n)=\sum_{d \mid n} d^{k}, \quad \sigma_{1}(n)=\sigma(n)
$$

It is well known that the coefficients in the expansion

$$
j(\tau)=\sum_{-1}^{\infty} c(n) x^{n}
$$

have remarkable divisibility properties. Lehner [7], [8] has shown, $a>0$,

$$
\begin{array}{ll}
c\left(2^{a} n\right) \equiv 0 & \left(\bmod 2^{3 a+8}\right), \\
c\left(3^{a} n\right) \equiv 0 & \left(\bmod 3^{2 a+3}\right), \\
c\left(5^{a} n\right) \equiv 0 & \left(\bmod 5^{a+1}\right) \\
c\left(7^{a} n\right) \equiv 0 & \left(\bmod 7^{a}\right) .
\end{array}
$$

The congruences (1.1) and (1.2) have been improved by Kolberg [1], [2]:

$$
\begin{gathered}
c\left(2^{a} n\right) \equiv-2^{3 a+8} 3^{a-1} \sigma_{7}(n) \quad\left(\bmod 2^{3 a+13}\right), \quad a \geqq 1, \quad n \text { odd } . \\
c\left(3^{a} n\right) \equiv \mp 3^{2 a+3} 10^{a-1} \sigma(n) / n \quad\left(\bmod 3^{2 a+6}\right) \\
\text { if } n \equiv \pm 1 \quad(\bmod 3) .
\end{gathered}
$$

Kolberg conjectured that (1.3) and (1.4) could be sharpened in a similar way, and in this note we shall deduce the following congruence

$$
c\left(5^{a} n\right) \equiv-3^{a-1} 5^{a+1} n \sigma(n) \quad\left(\bmod 5^{a+2}\right), \quad a>0 .
$$

Received July 1, 1964. 
Hence, especially

$$
c\left(5^{a}\right) \neq 0 \quad\left(\bmod 5^{a+2}\right),
$$

a conjecture of Lehner.

We shall also give a new proof of the congruence

$$
c(n) \equiv 10 n \sigma(n) \quad\left(\bmod 5^{2}\right), \quad(n / 5)=-1,
$$

where $(n / p)$ is Legendre's symbol. This congruence was proved by Kolberg in [3], where several other congruences for the modular invariant can be found.

2.

The following definitions and lemmas are all taken from Kolberg [4]. We put

$$
\begin{gathered}
\varphi(x)=\prod_{1}^{\infty}\left(1-x^{n}\right), \\
\Phi(x)=\varphi(x)^{k_{1}} \varphi\left(x^{2}\right)^{k_{2}} \ldots \varphi\left(x^{n}\right)^{k_{n}}, \quad k_{j} \text { integral . }
\end{gathered}
$$

Thus the symbol $\Phi(x)$ is not used to denote one particular function, its meaning will usually be different in different sections. A function of this form will be referred to as a $\Phi$-function.

Let $\Phi(x)=\Sigma P(n) x^{n}$ be the power series expansion of $\Phi(x)$. Further let $q$ be a given positiv integer. Then we put

It follows that

$$
\Phi_{j}=\sum P(q n+j) x^{q n+j}=\Phi_{j}\{\Phi(x)\} .
$$

$$
\Phi(x)=\Phi_{0}+\Phi_{1}+\ldots+\Phi_{q-1} ;
$$

we shall refer to this as the $q$-dissection of $\Phi(x)$. We define

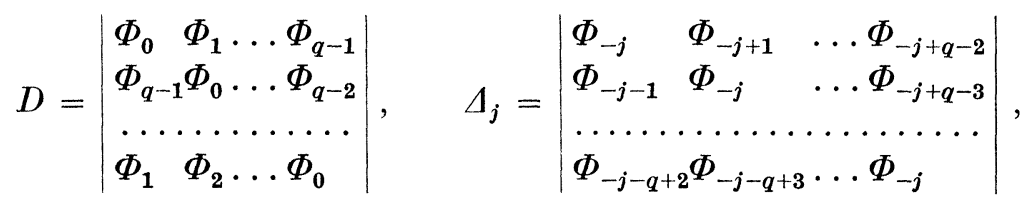

Thus $\Delta_{j}$ is the complement of $\Phi_{-j}$ in the circulant $D$.

Lemma. Let $q$ be a prime. Further, in the expression for $\Phi(x)$ let $k_{j}=0$ whenever $q \mid j$. Then we have

$$
D=\frac{\Phi\left(x^{q}\right)^{q+1}}{\Phi\left(x^{q^{2}}\right)} .
$$


Lemma. Let $\Phi(x)^{-1}=\Phi_{0}{ }^{\prime}+\Phi_{1}{ }^{\prime}+\ldots+\Phi^{\prime}{ }_{q-1}$ be the $q$-dissection of $\Phi(x)^{-1}$, where $\Phi(x)$ is an arbitrary $\Phi$-function. Then we have

$$
\Phi_{j}{ }^{\prime}=(-1)^{(q-1) j} D^{-1} \Delta_{j} .
$$

We shall use 5-dissection on $\varphi(x)$. From [5] we have

$$
\begin{gathered}
\varphi(x)=\varphi_{0}+\varphi_{1}+\ldots+\varphi_{4}, \\
\varphi_{3}=\varphi_{4}=0, \quad \varphi_{1}=-x \varphi\left(x^{25}\right), \quad \varphi_{0} \varphi_{2}=-\varphi_{1}{ }^{2},
\end{gathered}
$$

which follows from the well-known identities

$$
\begin{aligned}
\varphi(x) & =\sum_{-\infty}^{\infty}(-1)^{n} x^{1 / 2 n(3 n+1)} \quad \text { (Euler) }, \\
\varphi(x)^{3} & =\sum_{-\infty}^{\infty}(4 n+1) x^{n(2 n+1)} \\
& =\sum_{0}^{\infty}(-1)^{n}(2 n+1) x^{\frac{1}{2} n(n+1)} \quad \text { (Jacobi) } .
\end{aligned}
$$

In [5] Kolberg also proved

$$
\varphi_{0}^{5}+\varphi_{2}^{5}=\varphi\left(x^{5}\right)^{6} \varphi\left(x^{25}\right)^{-1}+11 x^{5} \varphi\left(x^{25}\right)^{5} .
$$

Elimination of $\varphi_{0}$ and $\varphi_{2}$ between (2.3) and (2.4) gives

$$
x \varphi\left(x^{5}\right)^{6} \varphi(x)^{-6}=V+5 V^{2}+15 V^{3}+25 V^{4}+25 V^{5},
$$

where $V=x \varphi(x)^{-1} \varphi\left(x^{25}\right)$. For the function $\varphi(x)^{3}$ we have

$$
\begin{gathered}
\varphi(x)^{3}=\left(\varphi_{0}+\varphi_{1}+\varphi_{2}\right)^{3}=\Phi_{0}+\Phi_{1}+\Phi_{3} . \\
\Phi_{0} \Phi_{1}=-3 x \varphi\left(x^{5}\right)^{6}-25 x^{6} \varphi\left(x^{25}\right)^{6}, \quad \Phi_{3}=+5 x^{3} \varphi\left(x^{25}\right)^{3} . \\
\Phi_{0}{ }^{5}+\Phi_{1}^{5}=\varphi\left(x^{25}\right)^{-3} \varphi\left(x^{5}\right)^{18}-9 \cdot 5^{2} x^{5} \varphi\left(x^{25}\right)^{3} \varphi(x)^{12}- \\
-9 \cdot 5^{4} x^{10} \varphi\left(x^{25}\right)^{9} \varphi\left(x^{5}\right)^{6}-11 \cdot 5^{5} x^{15} \varphi\left(x^{25}\right)^{15}
\end{gathered}
$$

Later on, when we write $\Phi_{0}, \Phi_{1}, \Phi_{3}$ it will always refer to the 5-dissection of $\varphi(x)^{3}$. We find it convenient to use the notation

$$
\varphi(x)^{6 k}=\Phi_{0 k}+\Phi_{1 k}+\ldots+\Phi_{4 k}, \quad \Phi_{j k}=\Phi_{j}\left\{\varphi(x)^{6 k}\right\} .
$$

3.

Our starting point is the following lemma (see Kolberg [6]): Let $p$ be one of the primes $2,3,5,7,13$ and put

$$
\Phi_{p}(\tau)=x\left(\varphi\left(x^{p}\right) / \varphi(x)\right)^{24 /(p-1)} .
$$


Then there exist constants $A_{k p}$ such that

$$
j(\tau)=\sum_{k=-1}^{p} A_{k p} \Phi_{p}(\tau)^{k}
$$

From this lemma we easily get the identity

$$
j(\tau)=f^{-1}+6 \cdot 5^{3}+63 \cdot 5^{5} f+52 \cdot 5^{8} f^{2}+63 \cdot 5^{10} f^{3}+6 \cdot 5^{13} f^{4}+5^{15} f^{5},
$$

where

$$
f=\Phi_{5}(\tau)=x \varphi\left(x^{5}\right)^{6} \varphi(x)^{-6} .
$$

We define the operator $L$ by

From (2.6) we get

$$
L \sum a_{n} x^{n}=\sum a_{5 n} x^{n} .
$$

$$
L f^{-1}=6-5^{2} f .
$$

If $q$ is a prime, we have the obvious congruence

$$
\varphi(x)^{q} \equiv \varphi\left(x^{q}\right) \quad(\bmod q),
$$

and using this and the well-known congruence (see [3])

$$
\sum_{1}^{\infty} n \sigma(n) x^{n} \equiv x \prod_{1}^{\infty}\left(1-x^{n}\right)^{24}(\bmod 5),
$$

we obtain from (3.1) and (3.2)

$$
\sum_{1}^{\infty} c(5 n) x^{n} \equiv-5^{2} \sum_{1}^{\infty} n \sigma(n) x^{n} \quad\left(\bmod 5^{3}\right) .
$$

The congruences refer to the coefficients of the power series in $x$. The last congruence (3.4) proves (1.7) for $a=1$.

To complete the proof of (1.7) it remains to show

$$
c\left(5^{a} n\right) \equiv 3^{a-1} 5^{a-1} c(5 n) \quad\left(\bmod 5^{a+2}\right) .
$$

To do so we shall obtain a formula for $L f^{k}, k>0$. By direct computation we find, using the results in section 2 ,

$$
\begin{aligned}
\Phi_{0}\{f\}= & x \varphi\left(x^{25}\right)^{6} \varphi\left(x^{5}\right)^{-30} \Delta_{-1} \\
= & 63 \cdot 5 x^{5} R^{6} S^{-6}+52 \cdot 5^{4} x^{10} R^{12} S^{-12}+63 \cdot 5^{6} x^{15} R^{18} S^{-18}+ \\
& +6 \cdot 5^{9} x^{20} R^{24} S^{-24}+5^{11} x^{25} R^{30} S^{-30},
\end{aligned}
$$

where

Hence

$$
R=\varphi\left(x^{25}\right), \quad S=\varphi\left(x^{5}\right) .
$$

$$
L f=63 \cdot 5 f+52 \cdot 5^{4} f^{2}+63 \cdot 5^{6} f^{3}+6 \cdot 5^{9} f^{4}+5^{11} f^{5} .
$$


Next, we will prove the following expressions for $L f^{k}, k=2,3,4$ :

$$
L f^{2}=\sum_{1}^{10} 5^{l} a f^{l}, \quad L f^{3}=\sum_{1}^{15} 5^{l-1} a f^{l}, \quad L f^{4}=\sum_{1}^{20} 5^{l-1} a f^{l} .
$$

Here, and in the following $a$ denotes an unspecified integer. From (2.2) we get

where

$$
\Phi_{0}\left\{f^{2}\right\}=x^{2} R^{12} S^{-60} \Delta_{-2},
$$

$$
\begin{aligned}
\Delta_{-2}=\Phi_{22} & -3 \Phi_{22}{ }^{2}\left(\Phi_{12} \Phi_{32}+\Phi_{02} \Phi_{42}\right)-\Phi_{02} \Phi_{12} \Phi_{32} \Phi_{42}+ \\
& +\Phi_{12}{ }^{2} \Phi_{32}{ }^{2}+\Phi_{02}{ }^{2} \Phi_{42}{ }^{2}+ \\
& +2 \Phi_{22}\left(\Phi_{02} \Phi_{32}{ }^{2}+\Phi_{12}{ }^{2} \Phi_{42}+\Phi_{32} \Phi_{42}{ }^{2}+\Phi_{02}{ }^{2} \Phi_{12}\right)- \\
& -\left(\Phi_{12} \Phi_{42}{ }^{3}+\Phi_{02} \Phi_{12}{ }^{3}+\Phi_{32} \Phi_{02}{ }^{3}+\Phi_{42} \Phi_{32}{ }^{3}\right)
\end{aligned}
$$

and where

$$
\begin{array}{ll}
\Phi_{02}=\Phi_{0}{ }^{4}+4 \Phi_{3}{ }^{3} \Phi_{1}+12 \Phi_{0} \Phi_{1}{ }^{2} \Phi_{3}, & \Phi_{32}=6 \Phi_{1}{ }^{2} \Phi_{3}{ }^{2}+4 \Phi_{1}{ }^{3} \Phi_{0}+4 \Phi_{0}{ }^{3} \Phi_{3} \\
\Phi_{12}=6 \Phi_{0}{ }^{2} \Phi_{3}{ }^{2}+4 \Phi_{0}{ }^{3} \Phi_{1}+4 \Phi_{1}{ }^{3} \Phi_{3}, & \Phi_{42}=\Phi_{1}{ }^{4}+4 \Phi_{3}{ }^{3} \Phi_{0}+12 \Phi_{0}{ }^{2} \Phi_{1} \Phi_{3} \\
\Phi_{22}=6 \Phi_{0}{ }^{2} \Phi_{1}{ }^{2}+12 \Phi_{0} \Phi_{1} \Phi_{3}{ }^{2}+\Phi_{3}{ }^{4} &
\end{array}
$$

We obtain

$$
\Delta_{-2}=\sum_{\substack{t, u \geq 0 \\ 5 u+2 t \leqq 16}} a \Phi_{0}{ }^{t} \Phi_{1}{ }^{t} \Phi_{3}{ }^{16-5 u-2 t}\left(\Phi_{0}^{5}+\Phi_{1}^{5}\right)^{u} .
$$

(2.7) and (2.8) yields

$$
\begin{gathered}
\Phi_{0}{ }^{t} \Phi_{1}{ }^{t}=\sum_{l=0}^{t} a 5^{2 t-2 l} x^{6 t-5 l} R^{6 t-6 l} S^{6 l}, \quad \Phi_{3}=5 x^{3} R^{3}, \\
\left(\Phi_{0}{ }^{5}+\Phi_{1}{ }^{5}\right)^{u}=R^{-3 u} S^{18 u}+a \cdot 5^{2} x^{5} R^{6-3 u} S^{18 u-6}+\sum_{v=2}^{3 u} a 5^{v+2} x^{5 v} R^{-3 u+6 v} S^{18 u-6 v} .
\end{gathered}
$$

Hence

$$
\begin{aligned}
\Phi_{0}\left\{f^{2}\right\} & =\sum_{\substack{t, u \geq 0 \\
5 u+2 t \leqq 16}} a \cdot 5^{16-5 u-2 t} x^{50-15 u-5 t} R^{60-18 u-6 t} S^{-60+18 u+6 t}+ \\
& +\sum_{\substack{t \geq 0 \\
u>0 \\
5 u+2 t \leqq 16}} a \cdot 5^{18-5 u-2 t} x^{55-15 u-5 t} R^{66-18 u-6 t} S^{-66+18 u+6 t}+ \\
& +\sum_{\substack{t \geq 0 \\
u>0 \\
5 u+2 t \leq 16 \\
3 u \geqq v \geqq 2}} a \cdot 5^{18-5 u-2 t+v} x^{50-15 u-5 t+5 v} R^{60-18 u-6 t+6 v} S^{-60+18 u+6 t-6 v} .
\end{aligned}
$$

We have thus got a polynomial in $f_{1}=f\left(x^{5}\right)$ of degree $\leqq 10$.

It remains to consider the exponent of 5 . We see at once that it is sufficient to consider the first of the three sums in the last expression for $\Phi_{0}\left\{f^{2}\right\}$. Putting 
Hence

$$
\begin{aligned}
& u=0, t=8 \text { we get } a f_{1}^{2} \text {, } \\
& u=0, t=7 \quad-\quad-\quad 5^{2} a f_{1}^{3} \text {, } \\
& u=0, t=6 \quad-\quad-\quad 5^{4} a f_{1}{ }^{4} \text {. }
\end{aligned}
$$

$$
L f^{2}=\sum_{l=1}^{3} a f^{l}+\sum_{4}^{10} 5^{l} a f^{l}
$$

We easily find

$$
\left\{\begin{array}{l}
L V=5 f \\
L V^{2}=2 \cdot 5 f+5^{3} f^{2} \\
L V^{3}=9 f+3 \cdot 5^{3} f^{2}+5^{5} f^{3} \\
L V^{4}=4 f+22 \cdot 5^{2} f^{2}+4 \cdot 5^{5} f^{3}+5^{7} f^{4}, \\
L V^{5}=f+20 \cdot 5^{2} f^{2}+40 \cdot 5^{4} f^{3}+5 \cdot 5^{7} f^{4}+5^{9} f^{5}, \\
L V^{6}=63 \cdot 5 f^{2}+52 \cdot 5^{4} f^{3}+63 \cdot 5^{6} f^{4}+6 \cdot 5^{9} f^{5}+5^{11} f^{6} .
\end{array}\right.
$$

Comparing now (2.5), (3.8) and (3.9) we obtain

$$
L f^{2}=\sum_{l=1}^{10} 5^{l} a f^{l} .
$$

By the same method we find

$$
L f^{3}=\sum_{1}^{4} a f^{l}+\sum_{5}^{15} 5^{l-1} a f^{l} .
$$

And again from (2.5), (3.9) and (3.10)

Similarly we find

$$
L f^{3}=\sum_{l=1}^{15} 5^{l-1} a f^{l} .
$$

$$
L f^{4}=\sum_{l=1}^{6} a_{l} f^{l}+\sum_{7}^{20} 5^{l-1} a f^{l} .
$$

The simplest way to investigate the six first coefficients in this case is to use the power series expansion of (3.11). Using Watson's table [10] of $\tau(n)$, and Newman's table [9] of $\eta(\tau)$, we find

We have thus proved (3.7).

$$
L f^{4}=\sum_{l=1}^{20} 5^{l-1} a f^{l} .
$$

From Lehner [7] we have

$$
\begin{aligned}
(3.12) f^{5}=f_{1} & +5 f\left(6 f_{1}+5^{2} f_{1}{ }^{2}\right)+5 f^{2}\left(63 f_{1}+6 \cdot 5^{3} f_{1}{ }^{2}+5^{5} f_{1}{ }^{3}\right)+ \\
& +5^{2} f^{3}\left(52 f_{1}+63 \cdot 5^{2} f_{1}{ }^{2}+6 \cdot 5^{5} f_{1}{ }^{3}+5^{7} f_{1}{ }^{4}\right)+ \\
& +5^{2} f^{4}\left(63 f_{1}+52 \cdot 5^{3} f_{1}{ }^{2}+63 \cdot 5^{5} f_{1}{ }^{3}+6 \cdot 5^{8} f_{1}{ }^{4}+5^{10} f_{1}{ }^{5}\right) .
\end{aligned}
$$


Noticing that $L f^{k} f_{1}^{s}=f^{s} L f^{k}$ we obtain from (3.6), (3.7) and (3.12)

$$
L f^{5}=\sum_{l=1}^{25} 5^{l-1} a f^{l} .
$$

By means of (3.6), (3.7), (3.12) and (3.13) we readily get the following general expressions for $L f^{k}, k>0$,

$$
\left\{\begin{array}{l}
L f^{5(k-1)+r}=\sum_{l=k}^{25(k-1)+5 r} 5^{l+1-k} a f^{l}, \quad r=1,2, \\
L f^{5(k-1)+p}=\sum_{l=k}^{25(k-1)+5 p} 5^{l-k} a f^{l}, \quad p=3,4,5 .
\end{array}\right.
$$

Defining

(3.14) implies

$$
T=\sum_{k=1}^{t} 5^{k} a f^{k}
$$

We obtain by (3.1) and (3.2)

(3.6) and (3.15) yields

$$
L j=744-5^{2} f+5^{3} T .
$$

and generally

$$
L^{2} j=744-(63) 5^{3} f+5^{4} T,
$$

$$
L^{a} j=744-(63)^{a-1} 5^{a+1} f+5^{a+2} T,
$$

which implies (3.5).

4.

Proof of (1.8). We put

$$
F=x \varphi(x)^{24} .
$$

5-dissection on (3.1) yields

$$
\begin{aligned}
& \sum c(5 n+2) x^{5 n+2}+\sum c(5 n+3) x^{5 n+3} \\
& \equiv \frac{1}{x} \varphi\left(x^{5}\right)^{-6}\left(\Phi_{3}\left\{\varphi(x)^{6}\right\}+\Phi_{4}\left\{\varphi(x)^{6}\right\}\right) \quad\left(\bmod 5^{2}\right) .
\end{aligned}
$$

We easily get

(4.1) and (4.2) give

$$
\begin{aligned}
\Phi_{3}\left\{\varphi(x)^{6}\right\}+\Phi_{4}\left\{\varphi(x)^{6}\right\} & =-10 \varphi_{1}{ }^{3}\left(\varphi_{0}{ }^{3}+\varphi_{2}{ }^{3}\right)-30 \varphi_{1}{ }^{4}\left(\varphi_{0}{ }^{2}+{\varphi_{2}}^{2}\right) \\
& \equiv 10 x^{3} \varphi(x)^{3} \varphi\left(x^{25}\right)^{3} \equiv 10 x^{3} \varphi(x)^{78} \quad\left(\bmod 5^{2}\right) .
\end{aligned}
$$

$$
\sum c(5 n+2) x^{5 n+2}+\sum c(5 n+3) x^{5 n+3} \equiv 10 F^{2}\left(\bmod 5^{2}\right) .
$$


We have

$$
x \varphi\left(x^{5}\right)^{4} \varphi(x)^{4} \equiv x \varphi(x)^{24} \quad(\bmod 5) .
$$

5-dissection on (3.4) yields

and

Hence

$$
\begin{aligned}
& \sum c(25 n+5) x^{5 n+1}+\sum c(25 n+20) x^{5 n+4} \\
& \equiv 10^{2} x \varphi\left(x^{5}\right)^{4}\left(\Phi_{0}\left\{\varphi(x)^{4}\right\}+\Phi_{3}\left\{\varphi(x)^{4}\right\}\right)\left(\bmod 5^{3}\right),
\end{aligned}
$$

$$
\begin{aligned}
\Phi_{0}\left\{\varphi(x)^{4}\right\}+\Phi_{3}\left\{\varphi(x)^{4}\right\} & =\varphi_{0}{ }^{4}+{\varphi_{2}}^{4}-8 \varphi_{1}{ }^{3}\left(\varphi_{0}+\varphi_{2}\right) \\
& \equiv \varphi(x)^{4}-x \varphi(x)^{28}(\bmod 5)
\end{aligned}
$$

(4.6) gives the following congruences:

$$
\Phi_{2}\{F\} \equiv \Phi_{2}\left\{F^{2}\right\}, \quad \Phi_{3}\{F\} \equiv \Phi_{3}\left\{F^{2}\right\} \quad(\bmod 5) .
$$

(3.3), (4.3) and (4.7) imply (1.8).

\section{REFERENCES}

1. O. Kolberg, Congruences for the coefficients of the modular invariant $j(\tau)$ modulo powers of 2, Univ. Bergen Årbok Naturvit. Rekke 1961, no. 16, pp. 9.

2. O. Kolberg, The coefficients of $j(\tau)$ modulo powers of 3, Univ. Bergen Årbok Naturvit. Rekke 1962, no. 16, pp. 7.

3. O. Kolberg, Congruences for the coefficients of the modular invariant $j(\tau)$, Math. Scand. 10 (1962), 173-181.

4. O. Kolberg, An elementary discussion of certain modular forms, Univ. Bergen Årbok Naturvit. Rekke 1959, no. 16, pp. 26.

5. O. Kolberg, Some identities involving the partition function, Math. Scand 5 (1957), 77-92.

6. O. Kolberg, Congruences for Ramanujan's function $\tau(n)$, Univ. Bergen Årbok Naturvit. Rekke 1962, no. 11, pp. 8.

7. J. Lehner, Divisibility properties of the Fourier coefficients of the modular invariant $j(\tau)$, Amer. J. Math. 71 (1949), 136-148.

8. J. Lehner, Further congruence properties of the Fourier coefficients of the modular invariant $j(\tau)$, Amer. J. Math. 71 (1949), 373-386.

9. M. Newman, A table of the coefficients of the powers of $\eta(\tau)$, Nederl. Akad. Wetensch. Indag. Math. (= Proc. Ser. A) 18 (1956), 204-216.

10. G. N. Watson, A table of Ramanujan's function $\tau(n)$, Proc. London Math. Soc. (2) 51 (1949), 1-13. 